\title{
Using Quantum Statistics to Win at Thermodynamics, and Cheating in Vegas
}

\author{
George S. Levy \\ Entropic Power, Irvine, CA, USA \\ Email: glevy@entropicpower.com
}

How to cite this paper: Levy, G.S. (2018) Using Quantum Statistics to Win at Thermodynamics, and Cheating in Vegas. Journal of Applied Mathematics and Physics, 6, 2166-2179.

https://doi.org/10.4236/jamp.2018.610182

Received: October 2, 2018

Accepted: October 28, 2018

Published: October 31, 2018

Copyright $\odot 2018$ by author and Scientific Research Publishing Inc. This work is licensed under the Creative Commons Attribution International License (CC BY 4.0).

http://creativecommons.org/licenses/by/4.0/

\begin{abstract}
Gambling is a useful analog to thermodynamics. When all players use the same dice, loaded or not, on the average no one wins. In thermodynamic terms, when the system is homogeneous-an assumption made by Boltzmann in his $\mathrm{H}$-Theorem-entropy never decreases. To reliably win, one must cheat, for example, use a loaded dice when everyone else uses a fair dice; in thermodynamics, one must use a heterogeneous statistical strategy. This can be implemented by combining within a single system, different statistics such as Maxwell-Boltzmann's, Fermi-Dirac's and Bose-Einstein's. Heterogeneous statistical systems fall outside of Boltzmann's assumption and therefore can bypass the second law. The Maxwell-Boltzmann statistics, the equivalent of an unbiased fair dice, requires a gas column to be isothermal. The Fermi-Dirac and Bose-Einstein statistics, the equivalent of a loaded biased dice, can generate spontaneous temperature gradients when a field is present. For example, a thermoelectric junction can produce a spontaneous temperature gradient, an experimentally documented phenomenon. A magnetic field parallel to, and an electric field perpendicular to a surface produce a spontaneous current along the surface, perpendicular to both fields (Reciprocal Hall Effect). Experimental data collected by several independent researchers is cited to support the theory.
\end{abstract}

\section{Keywords}

Entropy, Game, H-Theorem, Field-Induced Thermoelectric Effect, Reciprocal Hall Effect, Second Law, Thermodynamics, Thermoelectrics, Homogeneity, Indistinguishability

If the game is rigged, the best strategy is to cheat.

\section{Introduction}

Thermodynamics can be summarized as follows [1]: "You must play the game 
(zeroth law), you can't win, (first law), you can't break even (second law) and you can't quit the game (third law)." Obviously, the game is rigged. What to do? The only way to win is to cheat! In this context, the reader is asked to suspend the morally repugnant aspects of "cheating" and view it as stepping out of the box, a positive, rather than a negative and deceptive behavior. This article discusses the mathematics of "cheating" as statistical strategies aimed at breaking or bypassing the second law.

The second law of thermodynamics is one of the most respected and enduring in physics. Heat always flows from hot to cold. Entropy never decreases. Motion in isolated systems always comes to a stop. Friction rules. Perpetual motion machines capable of converting ambient heat to work are impossible. Quoting Einstein [1 Wikiquote]:

"It is the only physical theory of universal content which I am convinced will never be overthrown, within the framework of applicability of its basic concepts."

(Note Einstein's very circumspect underlined qualifier.)

Unlike conservation laws, the second law is statistical. A useful analogy is gambling in Vegas. In equivalence to friction, the house takes a cut, and it really does not matter whether a dice is loaded or not. When everyone plays with the same dice to equalize the odds, on the average, everyone loses. Of course, someone with a loaded dice can win if everyone else has a fair dice. But even though cheating does happen, this behavior is not permitted, and the cheater, if identified, is quickly kicked out of the casino. The statistics governing the game must be homogeneous, that is, the same for everyone. When no one cheats, everyone loses (on the average). This is the second law in Vegas.

The second law as currently proven, also requires homogeneous statistics throughout the system. In his H-Theorem, Boltzmann proves that entropy can never decrease [2]. Crucially he assumes a system comprised of homogeneously distributed particles [3] [4]. Gibbs, later, added the assumption of indistinguishability. Implied in these assumptions, is that all particles must follow the same statistics, in his case, the classical Maxwell-Boltzmann distribution. To be clear, Gibbs, in his paper "On the Equilibrium of Heterogeneous Substances", discusses several types of heterogeneous systems under the influence of gravity or the electromotive force, but he omits statistically heterogeneous systems. This omission is understandable given that in his time, late 1900's, gases were only known to follow the Maxwell-Boltzmann distribution. Quantum distributions such as Fermi-Dirac's and Bose Einstein were discovered in the 1920's.

When all components of a system are statistically homogeneous, (the same dice for everyone, loaded or not) the odds are equalized, and the second law is upheld.

Cheating, i.e., using a loaded dice when everyone else has a fair dice, is not permitted in Vegas-even though it does occur. This type of cheating can technically be described as a heterogeneous statistical strategy. Is cheating allowed in the physical world? In other words, can one step out of the box defined by 
Boltzmann's homogeneity assumption? Of course, mother nature enforces strictly impassable limits, but the question, as posed, is whether she allows heterogeneous statistical strategies. For this to be possible, the same system must include components with different statistics. In the gambling context, does nature allow players to cast different dice, fair or loaded, i.e., to cheat, regardless of casino rules.

Boltzmann never considered the possibility of multiple statistics in his proof. When he founded statistical thermodynamics in the nineteenth century, the Maxwell-Boltzmann distribution was the only known one suitable for describing the behavior of a gas. In other words, there was only one dice to play with. Thermodynamic cheating was not conceivable. With the advent of quantum mechanics, other distributions were discovered. Now fermions are known to comply with the Fermi-Dirac distribution and bosons with the Bose-Einstein distribution. These quantum statistics are the equivalent of loaded dice as they generate biased outcomes compared to the classical Maxwell-Boltzmann distribution and with each other. Another distribution of interest that also operates as a thermodynamic loaded dice is the biased half-maxwellian distribution. It arises at a surface in the presence of a magnetic field and an electric field [5].

Can these distributions be used to cheat and therefore get around the second law? Not when applied singly in statistically homogeneous systems, just like a single loaded dice does not change the odds when all players share it. Variants of the $\mathrm{H}$-Theorems for quantum systems have been developed by several researchers such as Von Neumann and Tolman [6] [7] [8], but, unfortunately just like Boltzmann, they assume statistical homogeneity which, from the outset, precludes cheating. This assumption is not always true in Vegas despite being a casino rule-and overly restrictive in the physical world, Boltzmann notwithstanding. All H-Theorems are widely and incorrectly believed to be "proofs" of the second law's universality when, in fact, they are indicators of its limitations.

The rest of this article describes possible implementations of heterogeneous statistical systems and discusses under what circumstances a temperature gradient arises in a gas column subjected to a downward force and whether this gradient can be used to break the second law. The following is a summary:

1) A Maxwellian gas with homogeneous statistics is always isothermal.

2) A non-Maxwellian gas with homogeneous statistics can produce a temperature gradient, but this gradient is not accessible to power a heat engine that converts ambient heat to useful energy.

3) Multiple non-maxwellian gases heterogeneously distributed in space and with different statistics can produce temperature gradients. These gradients can be used to generate energy, thereby bypassing the second law.

\section{Homogeneous Maxwellian Statistics-One Fair Dice Shared by Everyone}

One can begin the discussion with the conjecture by Johann Josef Loschmidt, a Boltzmann contemporary and a nineteenth century second law challenger. $\mathrm{He}$ 
asserted the following [9]:

1) A gas column such as air subjected to a vertical force such as gravity spontaneously develops a temperature gradient, and

2) A heat engine connected between the top and bottom of the column, could use this temperature gradient to convert ambient heat to useful energy thereby violating the second law.

As shall be explained below, Loschmidt was wrong, a maxwellian gas such as air cannot form a temperature gradient, as explained in [10]-[15] and summarized below. A gas that follows the Maxwell-Boltzmann statistics has the well-known kinetic energy distribution.

$$
f_{M B}\left(E_{k}\right)=2\left(\frac{1}{k_{B} \theta_{T}}\right)^{3 / 2} \sqrt{\frac{E_{k}}{\pi}} \exp \left(\frac{-E_{k}}{k_{B} \theta_{T}}\right)
$$

The derivation for the above equation can be found in [11]. If the gas is in a column, potential energy $E_{p}$ can be inserted to express the effect of elevation. The distribution then becomes,

$$
f_{M B}\left(E_{k}, E_{p}\right)_{\text {unnormalized }}=2\left(\frac{1}{k_{B} \theta_{T}}\right)^{3 / 2} \sqrt{\frac{E_{k}}{\pi}} \exp \left(\frac{-E_{k}-E_{p}}{k_{B} \theta_{T}}\right)
$$

This equation was derived from fundamental principles in [11]. The exponential term on the right is the Boltzmann factor. It expresses occupancy per microstate for a given total energy $E_{k}+E_{p}$. The square root term which stands for the density of states, (i.e., the number of microstates per velocity volume in phase space) is proportional to velocity, hence, to the square root of $E_{k}$ and is not a function of $E_{p}$. The remaining terms, in front, normalize the probability distribution which expresses occupancy as a function of kinetic energy.

The potential energy term can be factored out in its own exponential term as shown in the equation below:

$$
f_{M B}\left(E_{k}, E_{p}\right)_{\text {unnormalized }}=2\left(\frac{1}{k_{B} \theta_{T}}\right)^{3 / 2} \sqrt{\frac{E_{k}}{\pi}} \exp \left(\frac{-E_{k}}{k_{B} \theta_{T}}\right) \exp \left(\frac{-E_{p}}{k_{B} \theta_{T}}\right)
$$

Adding potential energy denormalized the distribution. Note that the distribution at elevation $Z$ corresponding to $E_{p}$ is scaled down by $\exp \left(-E_{p} / k_{B} \theta_{T}\right)$ :

$$
f_{M B}\left(E_{k}, E_{p}\right)_{\text {unnormalized }}=f_{M B}\left(E_{k}, E_{p}=0\right)_{\text {normalized }} \exp \left(\frac{-E_{p}}{k_{B} \theta_{T}}\right)
$$

which describes the well-known exponential density lapse with elevation, and confirms the validity of Equation (3).

To recover its normalized probabilistic form of Equation (1), one needs to renormalize Equation (3) by dividing it by $\exp \left(-E_{p} / k_{B} \theta_{T}\right)$. The renormalization process recovers Equation (1). Therefore, the distribution at any elevation is identical with the one at ground level and is therefore invariant with elevation. The gas column is isothermal and temperature $T_{1}$ and $T_{2}$ in Figure 1 are identical. 


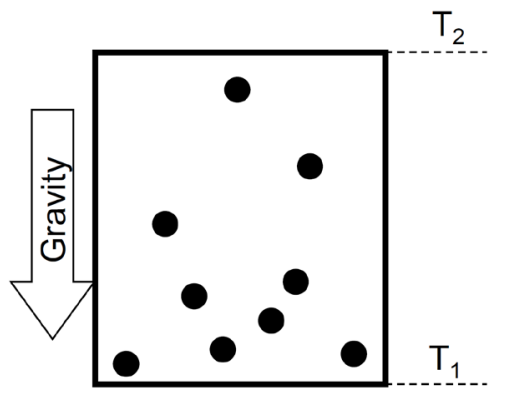

Figure 1. Maxwellian gas column subjected to a vertical force.

Interestingly, temperature can be expressed as an expectation of kinetic energy for a given distribution. For the Maxwell-Boltzmann distribution one can write:

$$
T\left(E_{p}, \theta_{T}\right)=\frac{2}{3 k_{B}} \int_{0}^{\infty} E_{k} f_{M B}\left(E_{k}, E_{p}, \theta_{T}\right)_{\text {normalized }} \mathrm{d} E_{k}
$$

Substituting (3) into (5) and solving the integral yields:

$$
T=\theta_{T}
$$

This equation is only true when the gas is maxwellian. Equations (5) and (6) express temperature in two ways: $\theta_{T}$ which is a parameter of the distribution, and $T$ which is proportional to the expected kinetic energy. Since $\theta_{T}$ is a parameter for the distribution that spans the whole column, $\theta_{T}$ is invariant with elevation-by definition. Furthermore, as per Equation (6), $T$ is also invariant with elevation (for a maxwellian gas), the column is isothermal as could have been predicted by Clausius version of the second law (heat flows from hot to cold and at equilibrium the temperature gradient is zero). A maxwellian gas column is isothermal with respect to both $\theta_{T}$ and $T$.

As we shall see in the next sections, depending on the distribution, $T=\theta_{T}$ is not always true which is the reason for using different symbols to differentiate between these two temperatures. The term thermodynamic temperature shall be avoided because of its heavy prejudicial baggage that fails to capture the difference between $T$ and $\theta_{T}$. Henceforth, $\theta_{T}$ shall be called statistical temperature because it is a parameter of the gas' statistics, and $T$, kinetic temperature, because it refers to the gas' kinetic energy. The next section covers fermions and bosons and describes situations in which $T$ and $\theta_{T}$ are unequal.

Loschmidt's assertion, as originally formulated was wrong. Breaking the second law with a maxwellian gas as he had originally conjectured, just like cheating by using a fair dice when everyone else also uses a fair dice, is not possible.

\section{Homogeneous Quantum Statistics-One Loaded Dice Shared by Everyone}

Would Loschmidt have been right had he formulated his conjecture using a non-maxwellian gas as shown in Figure 2? 


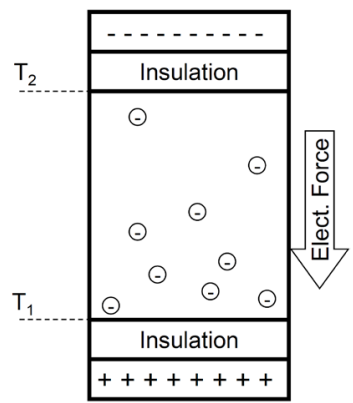

Figure 2. A fermion gas column, for example electrical carriers in a thermoelectric slab, subjected to a vertical electric field.

He would have been half right: 1) a temperature gradient would spontaneously develop but 2) no second law violation would occur. This is explained below.

The first half of Loschmidt's assertion that a temperature gradient develops is correct for the following reason. The distribution for a fermion gas in a column is:

$$
f_{\text {Fermions }}\left(E_{k}, E_{p}, \theta_{T}\right)_{\text {normalized }}=A\left(E_{p}, \theta_{T}\right) \sqrt{E_{k}} \frac{1}{1+\exp \left(\frac{E_{k}+E_{c}+E_{p}-E_{F}}{k_{B} \theta_{T}}\right)}
$$

The derivation of this equation from fundamental principle is available in [11]. The square root term represents the density of states which is solely a function of kinetic energy. As elevation increases, the insertion of potential energy in the Fermi-Dirac term denormalizes the distribution. In renormalization, however, $E_{p}$ cannot be factored out because potential energy is not expressed as a pure exponential as in a maxwellian gas. Therefore, this distribution, unlike the normalized Maxwell-Boltzmann distribution is not invariant with elevation.

Figure 3 illustrates the difference between the distributions. The thick red curve shows the distribution at ground level and the thin blue curve, at an arbitrary elevation. Maxwell-Boltzmann's distribution in 1) is invariant with elevation but Fermi-Dirac's in 2) is shifted to the left indicating a decrease in kinetic energy and a corresponding lowering of the kinetic temperature $T$. In other words, $T_{1}>T_{2}$, in Figure 1 .

Note that the statistical temperature $\theta_{T}$ is always invariant because it is a parameter of the distribution and therefore constant for a given ensemble spanning the column. Surprisingly, the gas is isothermal with respect to $\theta_{T}$ but not with respect to $T$. The same reasoning applies to a boson gas column.

The second half of Loschmidt's conjecture is that this temperature gradient can be used to produce useful energy (for example by thermally connecting a heat engine such as a Seebeck device between two points at different elevations). This assertion is incorrect.

As explained above, a gradient in kinetic temperature $T$ does occur spontaneously in the fermion column. However, the column remains isothermal with respect the statistical temperature $\theta_{T}$. This is confirmed by Tolman's proof ([6], 


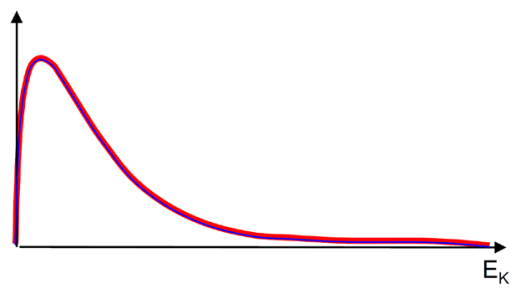

(a)

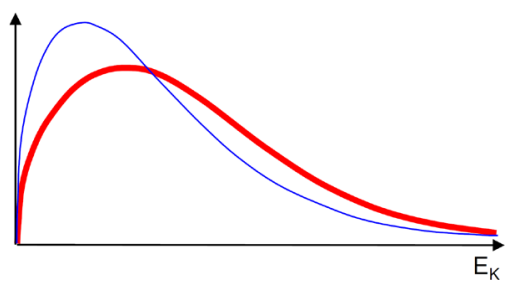

(b)

Figure 3. (a) Maxwell-Boltzmann energy distribution; (b) Fermi-Dirac energy distribution. The distributions for ground level are shown by the thick red curve and the one for a higher elevation, by the thin blue curve.

pages 551,552$)$ who shows that heat flows down the statistical temperature gradient, not necessarily the kinetic temperature gradient. Hence no heat flows along the column and the kinetic temperature gradient is not exploitable.

In the fully homogeneous system shown in Figure 4(a) the particles in the thermal connections are statistically identical with those in the column. Heat carriers in the thermal connections develop the same temperature gradient as those in the column. The temperature difference across the heat engine is zero, the engine cannot operate and. no work is produced.

An interesting analog is the built-in potential across a semiconductor junction as shown in Figure 4(b). The voltage across the leads to the junction exactly cancels the built-in potential. Hence, no voltage is detected by the voltmeter. Therefore, no energy can be extracted from a junction. A loop summation of voltages around the circuit yields zero because the electric field is conservative and scalar.

Both cases (the non-maxwellian gas column and the junction) conform to the $\mathrm{H}$-Theorem which assumes homogeneity and indistinguishability of particles. A voltage or a temperature gradient in a homogeneous system cannot be exploited for energy production. No cheating is possible when all players share the same dice, loaded or not.

\section{Heterogeneous Non-Maxwellian Statistics-Differently Loaded Dice for Different Players}

We can now formulate Loschmidt conjecture in a way that bypasses the second law. Two kinds of non-maxwellian statistics shall be discussed:

1) Heterogeneous Quantum statistics such as the Fermi-Dirac and Bose-Einstein distributions.

2) Biased half-maxwellian statistics generated near a surface by a magnetic and an electric field.

\subsection{Heterogeneous Quantum Statistics}

Consider the system shown in Figure 5.

Two different types of particles are present, each with its own susceptibility to the electric field and its own statistics. 


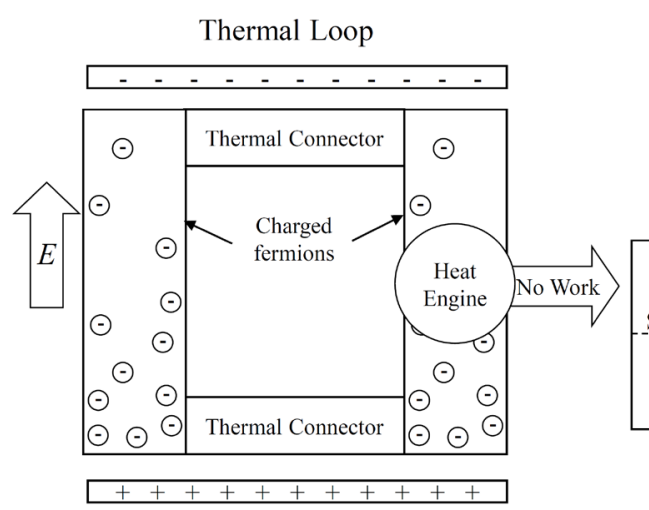

(a)
Electrical Loop

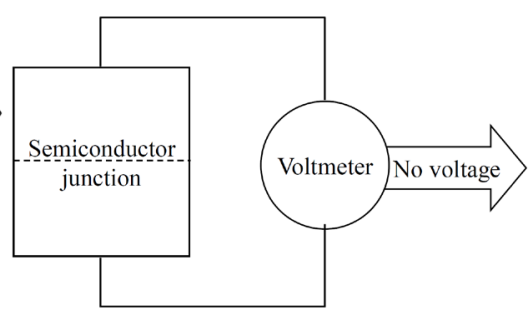

(b)

Figure 4. A homogeneous thermal system (a) comprising a non-maxwellian gas column with connections to a heat engine, is the analog of a junction (b) with leads connected to a voltmeter.

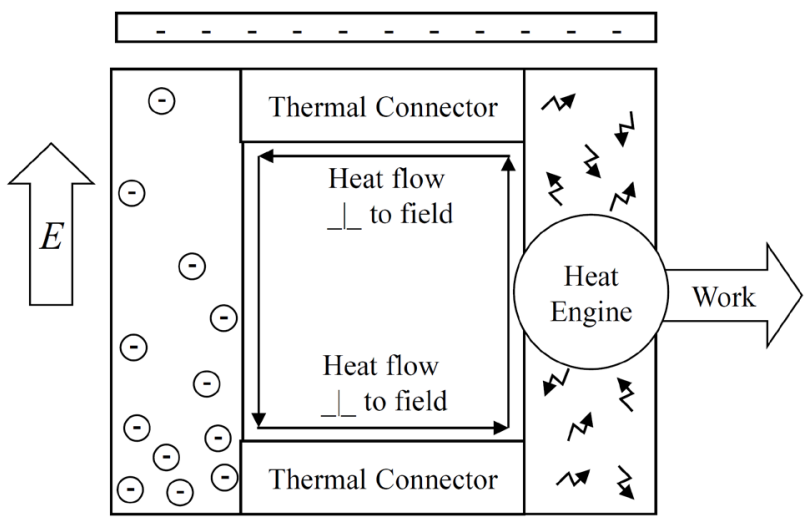

Figure 5. Statistically heterogeneous systems fall outside Boltzmann's assumption of homogeneity and can bypass the second law.

1) A column of charged fermions is subjected to a downward electrical field. The fermions could be embodied by electrical carriers in a slab of thermoelectric material between insulated capacitor plates. As explained above, in the section covering homogeneous quantum statistical systems, the fermions spontaneously develop a temperature difference.

2) Neutral bosons connect the top and the bottom of the columns to a heat engine. The bosons can be embodied by heat phonons in a thermal connector. The bosons are neutral. Therefore, they are not affected by the field, and do not spontaneously produce a temperature difference.

A net temperature difference results across the heat engine (for example a Seebeck device) thereby producing useful work.

How does this system get around the second law? Going back to the gambling analogy, two differently loaded dice are available, making cheating on the second law possible. Switching thermal carriers around the thermal loop, from charged fermions (electrons or holes, susceptible to the electric field), to uncharged bo- 
sons (thermal phonons, insensitive to the field) provides the loophole that gets around the limitation of the conservative field. One should note that Boltzmann's proof of the second law (his H-Theorem) relies on the assumption of homogeneity and indistinguishability of the particles. The statistically heterogenous system of Figure 5 falls outside of these assumptions, therefore bypassing the second law, not breaking it.

\subsection{Biased Half-Maxwellian Statistics}

Quantum mechanical distributions are not the only means of biasing statistics. In the Reciprocal Hall Effect [5], the velocity distribution of a charged gas, which is normally symmetrical in space, is skewed away from the normal to a surface in the presence of a magnetic field parallel to the surface and an electric field perpendicular to it, thereby generating a current. This effect is summarized below.

Consider low-density non-interacting charged gas particles, for example electrical carriers in a lightly doped thermoelectric slab, in contact with a surface, for example an insulator. The gas particles are thermalized by the surface, acquiring its distribution. If the distribution is maxwellian, then particles outgoing from the surface have a half-maxwellian distribution. After equilibrium is reached, incoming particles also possess a half-maxwellian distribution. Both distributions, outgoing and incoming, are symmetrical images of each other, and together they form a fully symmetrical maxwellian distribution.

If now a magnetic field $B$ is applied parallel to the slab, the particles are forced into partial cyclotron orbits interrupted by the surface as shown in Figure 6 thereby forming a current $I_{x}$ flowing on the inside surface of the slab. In the absence of electric field (to be discussed below) the current flows uniformly around the inside surface and is not observable from the outside.

If in addition to the magnetic field, an electric field $E_{z}$ is also applied perpendicular to the surface by means of capacitor plates, then the particles' trajectories become truncated cycloids. The electric field pushes the electrical carriers across the slab, causing depletion on one side, and excess carriers on the other side. This asymmetry results in a current pinch-off in the first side and an actual observable voltage or current on the second side as shown in Figure 7.

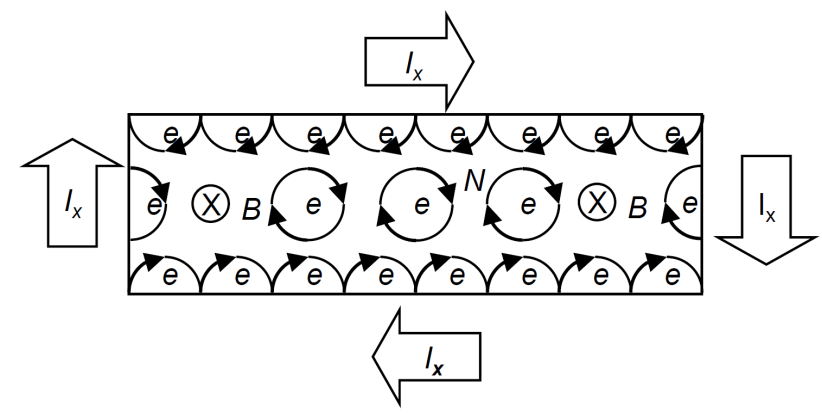

Figure 6. In the presence of a magnetic field in a slab of thermoelectric material, charged gas particles (electrical carriers) follow truncated cyclotron orbits interrupted by the surface of the slab. The resulting current inside the slab is not accessible from the outside. 


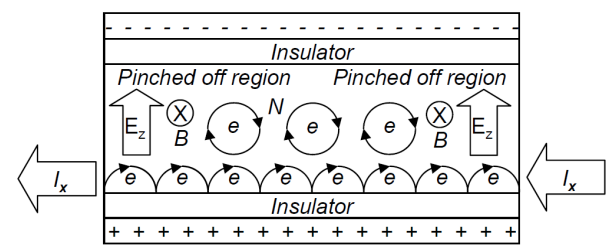

Figure 7. Reciprocal Hall Effect. A magnetic field parallel to a surface and an electric field perpendicular to the surface, cause a current perpendicular to both fields and along the surface.

Close examination of the particle statistics at the surface, shows that both half-maxwellian velocity distributions (for the incoming and outgoing particles) become biased in the same direction away from the normal to the surface. This biasing is shown in Figure 8 for a range of elevations.

The horizontal axis shows $4039 \mathrm{~m} / \mathrm{sec}$ per division. These distributions were generated by a publicly available program [14].

The Reciprocal Hall Effect is caused by statistical skewing of the particles' velocity distribution: in the presence of a magnetic field parallel to a surface and an electric field perpendicular to it, a current is generated along the surface perpendicular to both fields. (In contrast, the well-known Hall Effect generates an electric field perpendicular to a magnetic field and a current.) The statistical biasing is the analog of a loaded dice.

Can energy be extracted from such an effect? Not if the whole system including the leads is subjected to uniform electric and magnetic fields. As shown in Figure 5, the current would not be accessible from the outside and no energy would be produced. The system would be statistically homogeneous and covered by the H-Theorem.

Energy can only be produced by this effect if the system is statistically heterogeneous. In other words, the leads closing the current loop must be positioned outside the electric and magnetic fields, thereby ensuring that the velocity distribution of the carriers in the leads, remains symmetrical and that no Reciprocal Hall Effect occurs that would nullify the current.

\section{Experimental Data}

Several researchers in different labs have conducted experiments indicating violations of the second law. These experiments include:

\subsection{Unexplained Thermoelectric Phenomena}

Spontaneous kinetic temperature gradients are usually difficult to observe because they require unusual materials. For example, a semiconductor junction develops a temperature difference as well as a built-in potential. In a conventional semiconductor material, this temperature difference is quickly shorted by heat phonons traveling across the junction. However, it can be measured in high performance (ZT) thermoelectric materials which have a high thermal conductivity ratio between electrical carriers and thermal phonon. 


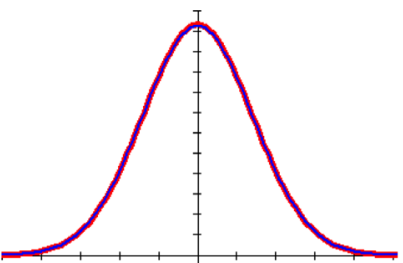

(a)

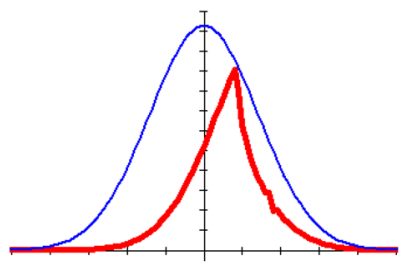

(c)

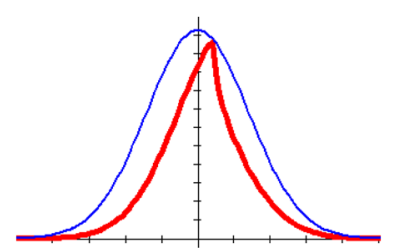

(b)

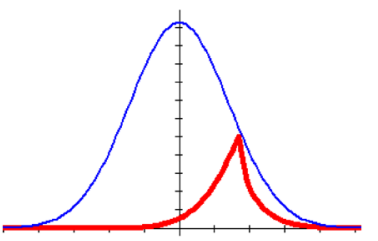

(d)

Figure 8. Horizontal velocity distribution of electrons caused by the reciprocal Hall Effect, in red at elevation (a) $0 \mathrm{~nm}$; (b) $100 \mathrm{~nm}$; (c) $200 \mathrm{~nm}$; (d) $400 \mathrm{~nm}$ above a surface for a magnetic field of 0.2 Tesla parallel to the surface. The blue curve provides a reference showing the distribution at zero elevation.

Researchers at Caltech performing measurements of the Seebeck coefficient on such materials observed mysterious data [15] [16]. Despite the most meticulous laboratory procedures they found that the voltage/temperature Seebeck curve did not go through the origin indicating a voltage output without a temperature difference input and a temperature difference output without any voltage input in apparent violation of the second law. The experiment (inadvertently) replicated the conditions outlined in this paper for this phenomenon to occur. The gas in the thermoelectric material was non-maxwellian (fermions) and subjected to a built-in potential within the semiconductor. The carriers in the measuring apparatus (temperature probe and electrical leads), were statistically different from those in the thermoelectric, thereby rendering the system statistically heterogeneous. This phenomenon can be called a field-induced thermoelectric effect and is unlike other well-known effects (Seebeck, Peltier and Thomson). It was described by the author at the International Thermoelectric Conference in Pasadena 2017 [15].

\subsection{Epicatalysis}

This phenomenon refers to a catalytic reaction that shifts the equilibrium state thereby departing from the behavior of a conventional catalyst which may speed the reaction but does not shift its equilibrium. It violates detailed balance and the second law because it allows continuous cycling between different equilibrium states with a continuous production of energy. This phenomenon is discussed by Levy [17] and has been experimentally verified in the lab (Sheehan [18] [19] [20] [21] [22]). Temperature differences of $126^{\circ} \mathrm{C}$ were observed between a tungsten catalyst and a rhenium catalyst operating on a hydrogen gas at $1950 \mathrm{~K}$. 
Experiments on epicatalysts have also been performed at room temperature by Miller [23]. He observed temperature differences in the order of $0.2 \mathrm{C}$ between catalysts operating on gases with weak hydrogen bonds such as formic acid.

\subsection{Q Machine}

This is a plasma device that also exhibits well documented epicatalytic behavior. [24] [25].

\section{Conclusions}

In the early days of thermodynamics-before Boltzmann-scientists had derived the second law by induction. No one had ever observed heat flowing from cold to hot or had constructed a perpetual motion machine of the second kind. Therefore, they concurred that the law must be true. Induction, however, is not reliable, as Bertrand Russel illustrates in the farmer/chicken paradox. A chicken fed every morning without fail, concludes using induction, that it is safe, but then, one day, its throat is wrung by the farmer.

Natural systems of particles with heterogeneous statistics are rare but they do exist. Their unusual behavior is usually masked by nearby homogeneous systems. As discussed in Section 5.1, the temperature gradient produced by electrical carriers in a semiconductor junction is quickly shorted by thermal phonons. However, it has been observed in high performance thermoelectrics, relatively rare materials.

Boltzmann provided a much needed deductive "proof" of the second law, that confirmed scientists inductively derived assertion. In his H-Theorem he makes the crucial assumption of a homogeneously distributed gas, thereby implying statistical homogeneity. His proof is over-restrictive as it covers homogeneous systems, not all possible physical systems including heterogeneous statistical systems. His proof highlights the limitation of the second law, not its universality.

Three examples of heterogeneous statistical systems capable of bypassing the second law have been described: 1) a temperature gradient produced by a field in a thermoelectric material and 2) a current produced by the combination of a surface, a magnetic field and an electric field (the Reciprocal Hall-Effect). 3) Epicatalysts. Experimental data from several independent researchers have been cited.

Cheating on mother nature is impossible, but cheating on man-made casino rules, or on the second law deduced using over-restrictive assumptions, may be forbidden but not impossible. Of course, the risk, in either case, is ending up with some broken ribs and/or a bruised reputation. However, with enough ingenuity, one can cheat on any laws devised by man that imperfectly portend to model nature. Heterogeneous statistical strategies fall outside these limits. Better understanding and appreciating the limits of the second law will revolutionize energy production and distribution. 
Paraphrasing Einstein's circumspect quote on the second law, such strategies do not fall "within the framework of applicability of its basic concepts."

Play fair, and what you lose in Vegas, stays in Vegas; cheat, and take your winnings out. Similarly, in thermodynamics, statistical homogenous strategies comply with the second law; heterogeneous statistical strategies bypass it and can be used to extract useful energy from ambient heat. But there is a difference. Cheating on the fair-play casino rules may leave you guilt-ridden; using heterogeneous statistical strategies to get around Boltzmann's over-restrictive assumption is exhilarating. When the cause is ethical and worthy, those strategies are simply another way to advance the common good, one step toward a cleaner, greener, environmentally-friendly future.

\section{Acknowledgements}

I would like to thank Daniel Sheehan for his encouragement, guidance and invaluable suggestions and my wife Penny for her unwavering support.

\section{Conflicts of Interest}

The authors declare no conflicts of interest regarding the publication of this paper.

\section{References}

[1] Wikiquote, Thermodynamics.

[2] Boltzmann, L. (1872) Weitere Studien über das Wärmegleichgewicht unter Gasmolekülen (Further Studies on the Thermal Equilibrium of Gas Molecules, The Kinetic Theory of Gases). Sitzungsberichte Akademie der Wissenschaften, 66, 275-370.

[3] Uffink, J. (2006) Compendium of the Foundations of Classical Statistical Physics. 44-45. http://philsci-archive.pitt.edu/2691/1/UffinkFinal.pdf

[4] Brown, H. and Myrvold, W. (2008) PhilSci-Archive. http://www.socsci.uci.edu/ bskyrms/bio/readings/brown_myrvold_on_boltzmann. pdf http://philsci-archive.pitt.edu/4187/1/Reversibility08.pdf

[5] Levy, G.S. (2017) Choosing between the Reciprocal Hall Effect, CPT Symmetry and the Second Law. The Open Science Journal of Modern Physics, 4, 1-8. https://www.researchgate.net/publication/314899020

[6] Tolman, R.C. (1938) The Principle of Statistical Mechanics, Oxford at the Clarendon Press.

https://archive.org/details/ThePrinciplesOfStatisticalMechanicsTolmanOxfordAtTh eClarendonPress1938

[7] Von Neumann, J. (1929) Proof of the Ergodic Theorem and the H-Theorem in Quantum Mechanics. Translator: Tumulka, R., arXiv:1003.2133v2, 27.

[8] Von Neumann, J. (1955) Mathematical Foundations of Quantum Mechanics. Princeton University Press, Princeton.

[9] Trupp, A. (1999) A. Energy, Entropy: on the Occasion of the 100th Anniversary of Loschmidt's Death in 1895: Is Loschmidt's Greatest Discovery Waiting for Its Discovery? Physics Essays, 12, 614-628. https://doi.org/10.4006/1.3028792 
[10] Levy, G.S. (2013) Thermoelectric Effects under Adiabatic Conditions. Entropy, 15, 4700-4715. https://doi.org/10.3390/e15114700

[11] Levy, G.S. (2015) Quantum Game Beats Classical Odds-Thermodynamics Implications. Entropy, 17, 7645-7657. https://doi.org/10.3390/e17117645

[12] Levy, G.S. (2016) Anomalous Temperature Gradient in Non-Maxwellian Gases. Proceedings of the 11 th International Conference on Ceramic Materials \& Components for Energy \& Environmental Applications, Vancouver, 14-19 June 2015, Vol. 255.

[13] Levy, G.S. (2017) Playing Rock, Paper, Scissors in Non-Transitive Statistical Thermodynamics. Journal of Applied Mathematics and Physics, 5, 1174-1197. https://doi.org/10.4236/jamp.2017.55102

[14] Levy, G.S. (2017) Reciprocal Hall Effect Calculator. http://www.entropicpower.com

[15] Levy, G.S. (2018) Temperature and Voltage Offsets in High ZT Thermoelectrics, International Conference on Thermoelectrics 2017. Journal of Electronic Materials, 47, 3067-3076. https://doi.org/10.1007/s11664-017-5875-0

[16] Iwanaga, S., Toberer, E.S., LaLonde, A. and Snyder, G.J. (2011) A High Temperature Apparatus for Measurement of the Seebeck Coefficient. Review of Scientific Instruments, 82, 1-6. https://doi.org/10.1063/1.3601358

[17] Levy, G.S. (2018) Quantum Physical Chemistry, the Law of Mass Action and Epicatalysis. http://www.entropicpower.com/technical-papers

[18] Sheehan, D.P. (2001) The Second Law and Chemically-Induced, Steady-State Pressure Gradients: Controversy, Corroboration and Caveats. Physics Letters A, 280, 185-190.

[19] Sheehan, D.P., Garamella, J.T., Mallin, D.J. and Sheehan, W.F. (2012) Steady-State Non-Equilibrium Temperature Gradients in Hydrogen Gas-Metal Systems: Challenging the Second Law of Thermodynamics. Physica Scripta, 2012, Article ID: 014030 .

https://www.researchgate.net/publication/258706086_Steady-state_nonequilibrium _temperature_gradients_in_hydrogen_gas-metal_systems_Challenging_the_second law_of_thermodynamics https://inis.iaea.org/search/search.aspx?orig_q=RN:44040484

[20] Sheehan, D.P. (2013) Nonequilibrium Heterogenous Catalysis in Long Term Mean-Free-Path Regime. Physical Review E, 88, Article ID: 032125. https://doi.org/10.1103/PhysRevE.88.032125

[21] Sheehan, D.P., Mallin, D.J., Garamella, J.T. and Sheehan, W.F. (2014) Experimental Test of a Thermodynamic Paradox. Foundations of Physics, 44, 235-247. https://doi.org/10.1007/s10701-014-9781-5

[22] Sheehan, D.P., Zawlacki, T.A. and Helmer, W.H. (2016) Apparatus for Testing Gas-Surface Reactions for Epicatalysis. Review of Scientific Instruments, 87, Article ID: 074101. https://doi.org/10.1063/1.4954971

[23] Miller, D.W. (2016) Apparatus and Procedures for Detecting Temperature Differentials with Epicatalytic Materials. http://associations.sou.edu/aaaspd/2016SANDIEGO/2016SympAbstracts/12.pdf

[24] Motley, R.W. (1975) Q-Machines. Academic Press, New York. https://doi.org/10.1016/B978-0-12-508650-9.50006-0

[25] Rynn, N. and D’Angelo, N. (1960) Device for Generating a Low Temperature, Highly Ionized Cesium Plasma. Review of Scientific Instruments, 31, 1326. 